\title{
Follicular fluid estradiol is an improved predictor of in vitro fertilization/intracytoplasmic sperm injection and embryo transfer outcomes
}

\author{
YAN LV ${ }^{1,2}$, SHENGYE DU ${ }^{3}$, XIN HUANG ${ }^{4,5}$ and CUIFANG HAO ${ }^{4,6}$ \\ ${ }^{1}$ Clinical Medical College of Shandong University, Jinan, Shandong 250012; \\ Departments of ${ }^{2}$ Gynecology and Reproductive Medicine, and ${ }^{3}$ Obstetrics, Jinan People's \\ Hospital, Jinan, Shandong 271100; ${ }^{4}$ Reproduction Medical Center, The Affiliated Yantai \\ Yuhuangding Hospital of Qingdao University, Yantai, Shandong 264000, P.R. China
}

Received September 29, 2018; Accepted August 20, 2019

DOI: $10.3892 / \mathrm{etm} .2020 .9256$

\begin{abstract}
The present study is a clinical trial analyzing follicular fluid. The current study aimed to assess whether a correlation exists among estradiol (E2), anti-Mullerian hormone (AMH) and prokineticin 1 (PROK1) levels in the follicular fluid. A total of 81 infertile patients (53 with primary infertility and 28 with secondary infertility) who received routine in vitro fertilization (IVF) and embryo transfer (ET) or intracytoplasmic sperm injection at Yuhuangding Hospital (Yantai, China) were included in the present study. On the day of egg retrieval, follicular puncture and follicular fluid extraction were performed on patients using double lumen needles under the guidance of a vaginal ultrasound. In 77 cases, follicular fluid was collected from the follicle with the largest diameter. A total of 53 cases underwent ET and subsequent pregnancy outcomes were traced. Concentrations of E2, AMH and PROK1 in the single follicular fluid specimens were determined. The concentration of E2 in follicular fluid from the largest follicles in absolute pregnancy group was significantly lower than that in absolute non-pregnancy group. The concentrations of PROK1 and AMH in follicular fluid from the largest follicles in absolute pregnancy group were not significantly different from those in absolute non-pregnancy group. The concentration of E2 was associated with the dosage of gonadotropin, but was not associated with age, AMH and
\end{abstract}

Correspondence to: Dr Cuifang Hao, Reproduction Medical Center, The Affiliated Yantai Yuhuangding Hospital of Qingdao University, 20 Yuhuangding East Road, Yantai, Shandong 264000, P.R. China E-mail: huangxin1887@163.com

Present addresses: ${ }^{5}$ Shanghai First Maternity and Infant Hospital, Tongji University School Medicine, Shanghai 201204; ${ }^{6}$ Department of Reproductive Medical Center, Qingdao Women and Children's Hospital, Qingdao, Shandong 266034, P.R. China

Key words: estradiol, anti-Mullerian hormone, prokineticin 1, follicular fluid, in vitro fertilization, embryo transfer
PROK1 levels in follicular fluid, fertilization rate or number of usable blastocysts. The area under curve revealed that E2 level in the follicular fluid exhibited a low predictive value for pregnancy outcome. The present study demonstrated that E2 level is a better predictor for the outcome of IVF-ET than AMH or PROK1 levels in the follicular fluid.

\section{Introduction}

The outcome of in vitro fertilization (IVF) is influenced by a number of factors. One of the most important factors is oocyte quality. The microenvironment of the follicular fluid is important for oocyte development (1). The analysis of oocyte-derived products, including follicular fluid and cumulus cells, has provided novel candidates that can be used to assess oocyte competence and embryo implantation ability (2-6). In the endometrium, prokineticin (PROK) 1 protein expression is highest at the midluteal phase of the menstrual cycle and this corresponds to the window of embryo implantation and has been reported to be a biomarker for human endometrial receptivity (7-9). PROKs promote the contractile function of ileal longitudinal muscles (10). PROK1 and PROK2 share $44 \%$ homology but exhibit different effects (10). PROK2 is mainly expressed in the central nervous system and in primary spermatocytes. PROK2 (-/-) mice display hypoplasia of olfactory bulb as well as an insufficient number and a reduced function of gonadotropin $(\mathrm{Gn})$ releasing hormone $(\mathrm{GnRH})$ neurons, which reduces GnRH secretion and affects the initiation of reproductive axis, which is related to the occurrence of Kallmann Syndrome (11). Additionally, PROK2 plays an important role in the final stage of spermatogenesis (12). The selection of dominant follicles often requires more complex capillary networks and angiogenesis is closely associated with follicular development (13). PROK1 has been revealed to promote angiogenesis and is also known as endocrine gland (EG)-derived vascular endothelial growth factor (VEGF) (10). The PROK1 precursor protein contains 19 signal peptides that are formed by amino acids (AA). Mature PROK1 is composed of 86 AA but does not belong to the VEGF family. However, mature PROK1 shares homology with mamba intestinal toxin 
and bombina variegate 8 (14). Human PROK1 is expressed mainly in the glands that produce natural steroids (10,14-19). Kisliouk et al (18) demonstrated that the PROK mRNA was expressed in luteinizing granulosa cells. Treatment with denylate cyclase activator forskolin was revealed to upregulate PROK1 mRNA expression in SVOG cells in a dose-dependent manner (18). By contrast, classical angiogenic factors, including hypoxia and thrombin, inhibit PROK1 expression in SVOG cells (18). Studies have indicated that PROK1 expression in human, chimpanzee and bovine ovaries exhibited clear spatial and temporal specificity $(8,14,18)$. It has also been revealed that PROK1 is dynamically distributed during folliculogenesis, meaning that the expression of PROK1 in primordial and primary follicles is significantly higher compared with expression in the antral follicles (19). This suggests that the differential regulation of PROK1 expression by hormones exists during follicular maturation (19). It has been revealed that human chorionic Gn (HCG) can increase PROK1 expression in luteinizing granulosa cells and the placenta by binding with luteinizing hormone/choriogonadotropin receptor (20). This indicates that the increase in PROK1 secretion and expression is a response to peaks in physiological luteinizing hormone ( $\mathrm{LH})$ and that exogenous HCG and is an important factor in the final maturation of oocytes (20). A previous study has demonstrated that the level of PROK1 in the follicular fluid of the implantation group was significantly higher compared with the non-implantation group. Implantation potential could also be predicted if PROK1 >5.7 ng/follicle (19). Furthermore, it has also been reported that no significant difference in PROK1 level in the follicular fluid was observed between the pregnant group with a normal ovarian response and the non-pregnant group with a normal ovarian response (21). Anti-Mullerian hormone $(\mathrm{AMH})$ is a glycoprotein in the transforming growth factor- $\beta$ superfamily and is secreted by granular cells in the sinusoidal and preantral follicles (22). After HCG injection, the granular cells are highly luteinized and the secretion of AMH may therefore be reduced (23). It remains undetermined whether changes in follicular fluid or peripheral blood PROK1 during an ovulation induction cycle are closely associated with vascularization and follicular development and if this can represent the degree of follicle maturation or predict pregnancy outcome. In the present study, PROK1, AMH and estradiol (E2) was assessed in follicular fluid and the present study aimed to assess if these can predict the outcome of clinical pregnancy. Assessments were performed using follicular fluid secreted by highly luteinizing granulosa cells during the induction of ovulation. The current study also aimed to assess whether there was a correlation among E2, AMH and PROK1 levels in in follicular fluid and whether E2, AMH and PROK1 levels were associated with fertilization rate, good embryo rate and the number of usable blastocysts, which indirectly reflected the potential success rate of embryo implantation.

\section{Materials and methods}

Patients. A total of 81 infertile female patients (53 with primary infertility and 28 with secondary infertility) who received routine IVF-ET or intracytoplasmic sperm injection (ICSI) between May and July 2016 at the Reproductive Medical Center of the Yuhuangding Hospital (Yantai,
China) were included in the present study. The mean age of the patients was $32.30 \pm 3.67$ years, mean body mass index (BMI) was $24.05 \pm 3.98 \mathrm{~kg} / \mathrm{m}^{2}$, infertile period was $4.57 \pm 2.8$ years, basal follicle stimulating hormone (FSH) level was $6.56 \pm 2.16 \mathrm{mIU} / \mathrm{ml}$ and basal AMH level prior to downregulation was $4.12 \pm 2.98 \mathrm{ng} / \mathrm{ml}$. Among all patients included in the current study, 63 underwent routine IVF (including 1 patient who received a sperm donation), 15 underwent ICSI and 3 underwent IVF + rescue ICSI. Patients aged $>45$ years, patients who exhibited karyotype abnormalities, had a history of ovarian hyperstimulation syndrome (OHSS), endometriosis, repeated embryo implantation failures, ectopic pregnancy, ovarian surgery, ovarian benign or malignant tumors or smoking were excluded from the present study. No eligible follicular fluid was obtained from 4 of the patients and the oocytes were not mature after ovum retrieval in 2 patients. A total of 75 cases underwent IVF/ICSI and embryo culture. Among these 75 cases, 22 cases cancelled the embryo transfer. As a result, a total of 53 cases underwent embryo transfer and their pregnancy outcomes were traced. All procedures performed in the current study were approved by the Ethics Committee of Yuhuangding Hospital. Written informed consent was obtained from all patients or their families.

Downregulation, ovulation induction and follicular monitoring. Among the 81 patients, 62 received downregulation using a long regimen with luteal phase $\mathrm{Gn}$ releasing hormone (GnRH) agonist (24). A total of 7 days after ovulation, $0.05 \mathrm{mg}$ triptorelin (Ipsen Pharma Biotech S.A.S.) was given to patients for a period of 16 days of downregulation. The starting dose and timing of $\mathrm{Gn}$ were subsequently determined according to the number of antral follicles, body mass index and size of follicles in patients $(25,26)$. Transvaginal ultrasound scans were performed on day 6 of ovarian stimulation. Follicle diameters $<10 \mathrm{~mm}$ and E2 levels $<200 \mathrm{pg} / \mathrm{ml}$ on day 6 were defined as a slow response. If the slow response occurred, the dose of Gn was increased. Gn included recombinant human follicle stimulating hormone (Gonal- $\mathrm{F}^{\circledR}$; Merck KGaA) and human menopausal Gn (Lizhu Pharmaceutical Trading Co., Ltd.). Transvaginal ultrasound scan was performed on day 6 of ovarian stimulation. If the low response occurred, the dose of Gn needed to be increased. The remaining 19 patients underwent GnRH-antagonist regimen (27). During the menstrual period, a transvaginal ultrasound was used to confirm that no follicle exceeded $10 \mathrm{~mm}$ in diameter. According to the ovarian reserve, body mass index and follicle size, the starting dose of Gn was determined (26). Patients with follicle diameters $>14 \mathrm{~mm}$ or luteinizing hormone $(\mathrm{LH})$ expression levels $>10 \mathrm{mIU} / \mathrm{ml}$, received injections of $0.25 \mathrm{mg}$ ganirelix acetate (Orgalutran; Merck Sharp \& Dohme-Hoddesdon) prior to the injection of human chorionic Gn (HCG). The time of HCG injection (8000 IU; Lizhu Pharmaceutical Trading Co., Ltd.) was determined by follicular size (the diameter of at least 2 dominance follicles $\geq 18 \mathrm{~mm}$ ) and levels of E2 (at least $300 \mathrm{pg} / \mathrm{ml}$ per dominant follicle) (28).

Oocyte retrieval and fertilization. At 34-36 h after HCG injection, oocytes were retrieved under the guidance of a vaginal ultrasound. Oocytes were hatched $4 \mathrm{~h}$ prior to in vitro fertilization. Embryos were transferred on day 3 (cleavage 
Table I. Baseline characteristics of enrolled patients.

Characteristic

Statistics, median (interquartile range)/mean $\pm \mathrm{SD} / \%$ rate

Gn dosage (IU)

E2 on HCG day (pg/ml)

Endometrium thickness on HCG day (mm)

E2 concentration in peripheral blood on day of OR $(\mathrm{pg} / \mathrm{ml})$

PROK 1 concentration in peripheral blood on day of OR $(\mathrm{pg} / \mathrm{ml})$

AMH concentration in peripheral blood on day of OR $(\mathrm{ng} / \mathrm{ml})$

Follicular fluid specimen volume (ml)

E2 concentration in follicular fluid $(\mathrm{pg} / \mathrm{ml})$

AMH concentration in follicular fluid ( $\mathrm{ng} / \mathrm{ml})$

PROK1 concentration in follicular fluid $(\mathrm{pg} / \mathrm{ml})$

No. of retrieved oocytes

No. of mature oocytes

No. of fertilized oocytes

Good embryo rate

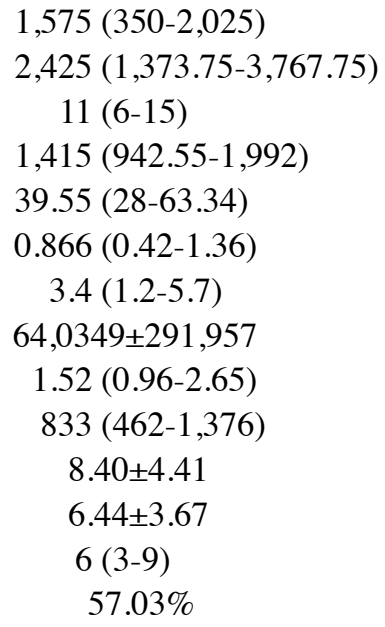

FSH, follicle-stimulating hormone; AMH, anti-Mullerian hormone; Gn, gonadotropin; E2, estradiol; HCG, human chorionic Gn; PROK1, prokineticin 1; OR, oocyte retrieval; SD, standard deviation. Data with normal distribution are expressed as the mean \pm SD. Data with non-normal distribution were expressed using the median (25-75\%).

Table II. Comparison of variables between absolute pregnancy group and non-pregnancy group.

\begin{tabular}{|c|c|c|c|c|}
\hline Variable & $\begin{array}{l}\text { Absolute pregnancy } \\
\text { group }(n=21)\end{array}$ & $\begin{array}{l}\text { Absolute non-pregnancy } \\
\text { group }(n=27)\end{array}$ & $\begin{array}{l}\text { Statistical } \\
\text { value }\end{array}$ & P-value \\
\hline Age (years) & $32.29 \pm 2.59$ & $32.26 \pm 4.27$ & $0.027^{\mathrm{a}}$ & 0.979 \\
\hline Years of infertility & $4.95 \pm 3.09$ & $4.87 \pm 2.98$ & $0.093^{\mathrm{a}}$ & 0.926 \\
\hline Constituent ratio of primary infertility & $47.62 \%(10 / 21)$ & $70.37 \%(19 / 27)$ & $2.557^{\mathrm{b}}$ & 0.110 \\
\hline Body mass index $\left(\mathrm{kg} / \mathrm{m}^{2}\right)$ & $24.84 \pm 3.94$ & $23.87 \pm 3.41$ & $0.909^{\mathrm{a}}$ & 0.368 \\
\hline Basal FSH (mIU/ml) & $6.54(2.95-10.04)$ & $6.56(3.25-12.45)$ & $-0.753^{c}$ & 0.451 \\
\hline Basal AMH (ng/ml) & $4.17 \pm 2.69$ & $3.67 \pm 2.25$ & $0.707^{\mathrm{a}}$ & 0.483 \\
\hline Gn dosage (IU) & $1,855 \pm 421$ & $1,628 \pm 448$ & $1.797^{\mathrm{a}}$ & 0.079 \\
\hline E2 on HCG day (pg/ml) & $2,232 \pm 1,317$ & $2,640 \pm 1,974$ & $0.108^{\mathrm{a}}$ & 0.421 \\
\hline Endometrium thickness on HCG day (mm) & $11(9-12)$ & $7(4.25-10.75)$ & $-0.274^{c}$ & 0.784 \\
\hline No. of retrieved oocytes & $9.00 \pm 3.45$ & $7.28 \pm 3.56$ & $1.789^{\mathrm{a}}$ & 0.079 \\
\hline E2 concentration on day of OR $(\mathrm{pg} / \mathrm{ml})$ & $1,415(985-1,673)$ & $1,199(718-2336)$ & $0.322^{\mathrm{c}}$ & 0.747 \\
\hline PROK1 concentration on day of OR (pg/ml) & $44.33(16-285)$ & $41.03(13-314)$ & $-1.091^{\mathrm{c}}$ & 0.275 \\
\hline AMH concentration on day of OR (ng/ml) & $0.798(0.15-4.14)$ & $0.835(0.66-4.8)$ & $-0.200^{c}$ & 0.841 \\
\hline PROK1 concentration in follicular fluid ( $\mathrm{pg} / \mathrm{ml})$ & $797.91(235.99-2,653.33)$ & $836.78(201.61-7,468.00)$ & $-0.785^{\mathrm{c}}$ & 0.433 \\
\hline AMH concentration in follicular fluid (ng/ml) & $1.63(0.47-6.96)$ & $1.27(0.31-13.69)$ & $-1.232^{c}$ & 0.218 \\
\hline E2 concentration in follicular fluid (pg/ml) & $526,419 \pm 289,944$ & $736,085 \pm 261,885$ & $-2.626^{\mathrm{a}}$ & 0.012 \\
\hline Fertilization rate & $88.4 \%(137 / 155)$ & $78.5 \%(146 / 186)$ & $5.861^{\mathrm{b}}$ & 0.015 \\
\hline No. of usable blastocysts & $2.62 \pm 2.31$ & $1.58 \pm 2.04$ & $1.596^{\mathrm{a}}$ & 0.118 \\
\hline
\end{tabular}

stage) or day 5/day 6 (blastocyst stage). Only fertilized oocytes (2 pronuclei; $2 \mathrm{PN}$ ) were further cultured to the blastocyst stage for 5 or 6 days, and unfertilized oocytes were discarded. Luteal support was provided after transplantation. Progesterone capsules (200 mg; UTROGESTAN; Besins Healthcare) were given via vaginal administration, $\mathrm{q} 8 \mathrm{~h}$, until week 10 of gestation. Transplantation was cancelled if (OHSS) occurred, the thickness of endometrium was $<7 \mathrm{~mm}$ or no transplantable 
A

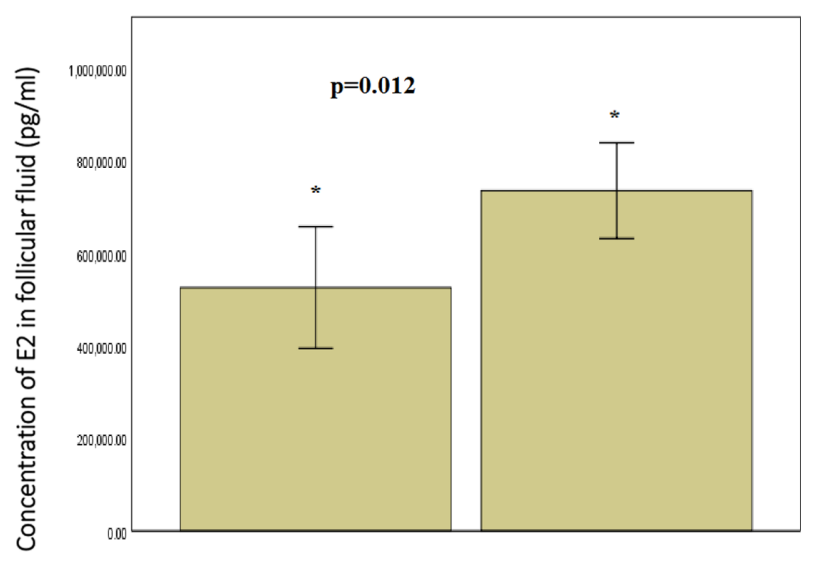

Absolute pregnancy Absolute non-pregnancy

B

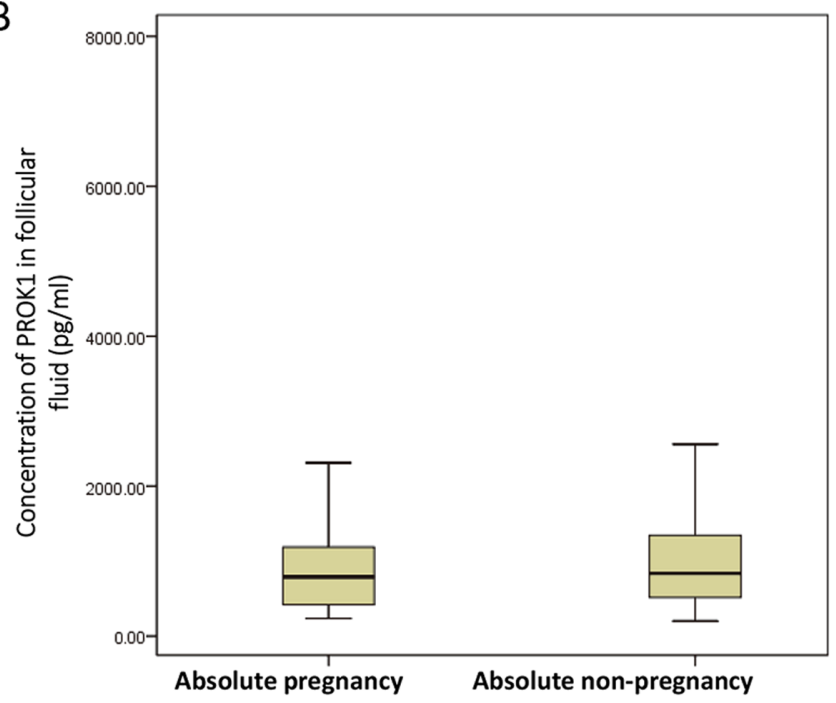

C

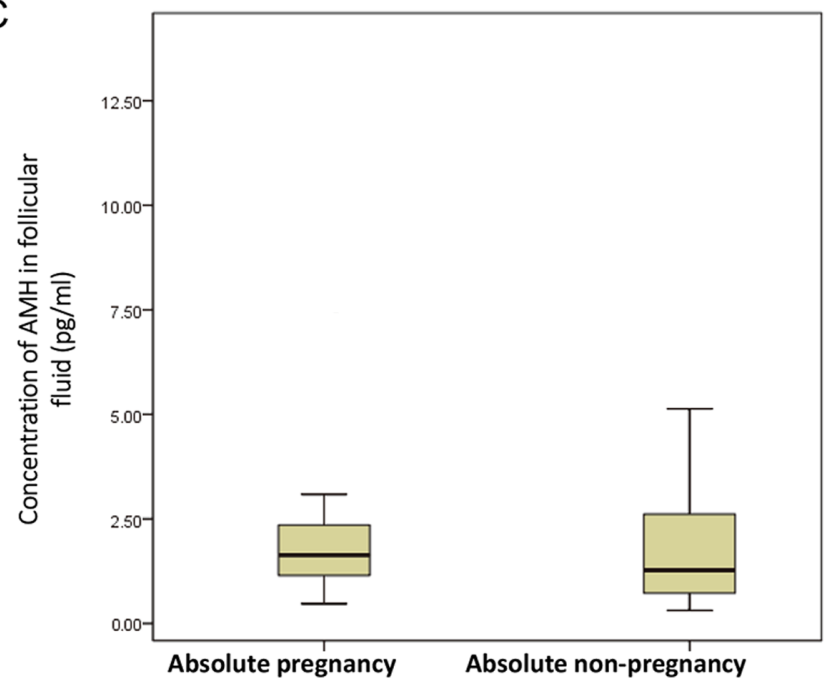

Figure. 1. Concentrations of (A) E2, (B) PROK1 and (C) AMH in follicular fluid in absolute pregnancy group and absolute non-pregnancy group. The results in (A) are presented as the mean \pm SD. The results in (B) and (C) are presented as box-plot diagrams (These are split up by the maximum-upper quartile-median-lower quartile-minimum). ${ }^{*} \mathrm{P}<0.05$. E2, estradiol; PROK1, prokineticin 1; $\mathrm{AMH}$, anti-Mullerian hormone; SD, standard deviation

embryo was available. The total fertilization rate of IVF was calculated using the following formula: $\mathrm{N}=$ number of $2 \mathrm{PN}$ oocytes + number of 1PN oocytes + number of multi-PN oocytes + number of 0PN cleavage oocytes)/total number of sperm oocytes x100. Total fertilization rate of ICSI was calculated using the following formula: $\mathrm{N}=$ (number of $2 \mathrm{PN}$ oocytes + number of 1PN oocytes + number of multi-PN oocytes)/total number of oocytes injected with MetaphaseII $\mathrm{x} 100$. These results were combined together to get the overall fertilization rate, The formula for calculating the good embryos rate is as follows: Good embryo rate=the number of good embryos/the number of cleavage stage embryos. Cleavage embryos with seven or eight cells on day 3 after oocyte retrieval that contained $<20 \%$ anucleate fragments and no apparent morphological abnormalities were classified as good embryo (29).

Sample collection. On the day of egg retrieval, follicular puncture and follicular fluid extraction were performed on patients using double lumen needles under the guidance of a vaginal ultrasound. Follicular fluid was collected from the follicle with the largest diameter (single follicular fluid) prior to centrifugation at $3,000 \times \mathrm{g}$ for $15 \mathrm{~min}$ at $4^{\circ} \mathrm{C}$ to separate cells. Fasting elbow vein blood $(2 \mathrm{ml})$ was collected in anticoagulant blood tubes on the morning of egg retrieval. The blood was centrifuged at $450 \mathrm{x} \mathrm{g}$ for $30 \mathrm{~min}$ in $25^{\circ} \mathrm{C}$ prior to serum collection.

Grouping standard. A total of 2 weeks after transplantation, patients with blood $\beta-\mathrm{HCG}<10 \mathrm{mIU} / \mathrm{ml}$ were included in the absolute non-pregnancy group $(n=27)$. A total of 34 days after transplantation, patients with blood $\beta$-HCG $>10 \mathrm{mIU} / \mathrm{ml}$ received ultrasonography and those with a gestational sac were included in the absolute pregnancy group $(n=20)$. If blood $\beta$-HCG was $>10 \mathrm{mIU} / \mathrm{ml}$ but no gestational sac was observed, the patients were included in the possible pregnancy group $(n=6)$, which was not included in the analysis of the current study.

Electrochemiluminescence. A Cobas e Immunoassay Analyzer (Roche Diagnostics) was used to determine E2 and $\beta-\mathrm{HCG}$ levels in the follicular fluid according to the manufacturer's protocol. The dilution ratio of follicular fluid ranged from 1:100 to 1:400. The lower and upper limits of E2 detection were $18.4 \mathrm{pmol} / \mathrm{l}(5 \mathrm{pg} / \mathrm{ml})$ and 11,010 pmol/l $(3,000 \mathrm{pg} / \mathrm{ml})$, respectively.

ELISA. The levels of PROK1 in serum and follicular fluid were determined using a PROK1 ELISA kit (cat. no. 900-k244; PeproTech Inc.), and the detection concentration range was $6-2000 \mathrm{pg} / \mathrm{ml}$. The sample was diluted if the concentration was out of the aforementioned range. AMH level in both serum and follicular fluid. was determined using an AMH ELISA kit (cat. no. KR-001; Guangzhou Kangrun Biotech Co., Ltd.) according to manufacturer's protocol. The detection range was 0.06-18 ng/ml.

Statistical analysis. All results were analyzed using SPSS software (version 21.0; IBM Corp.). Each measurement was repeated three times. The means of two groups of data with a normal distribution were compared using a Student's t-test. A Mann-Whitney U rank sum test was used to compare the difference between two groups of non-normal distribution. 
A

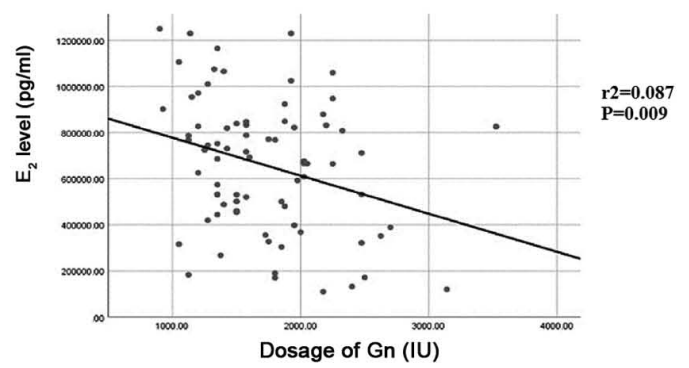

C



$\mathbf{E}$

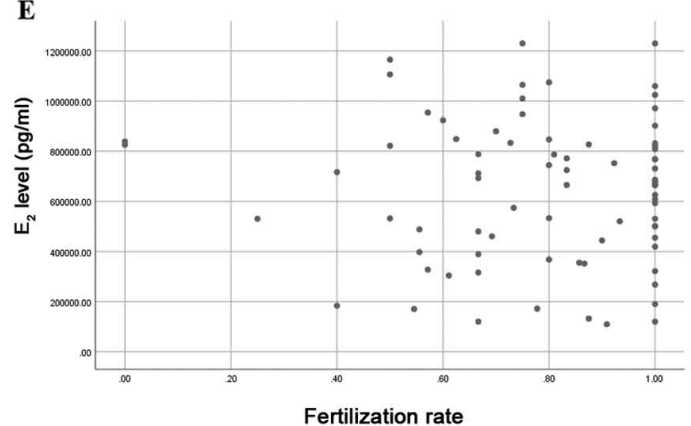



D

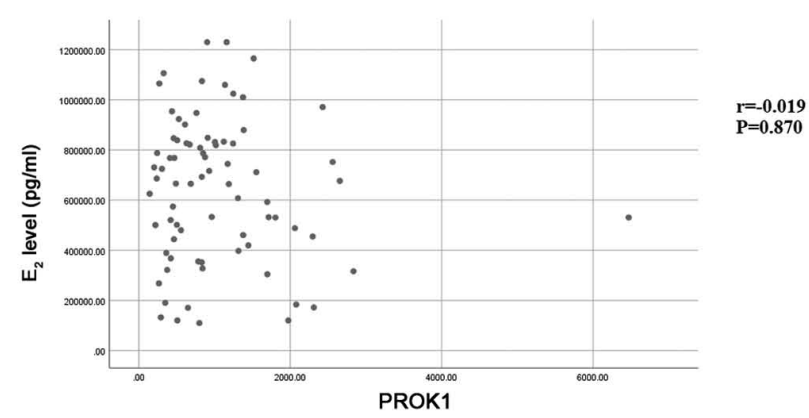

F

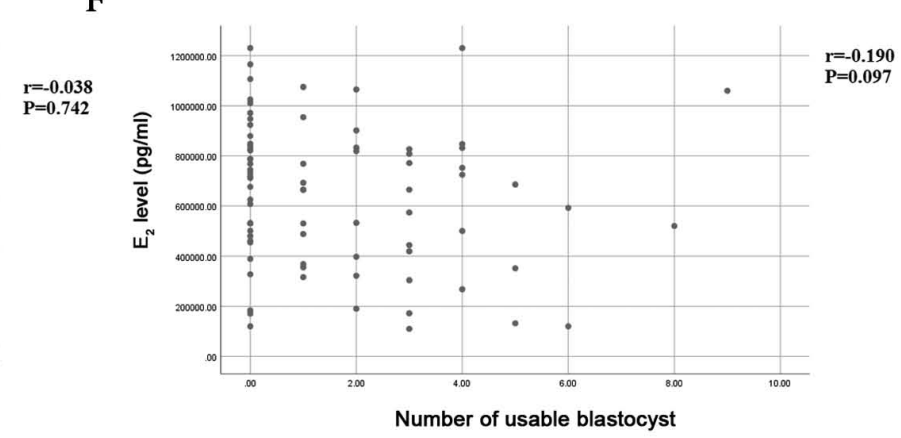

Figure. 2. Association between the E2 concentration in follicular fluid and (A) Gn dosage, (B) age, (C) AMH in follicular fluid, (D) PROK1 in follicular fluid, (E) fertilization rate and (F) the number of usable blastocysts. E2, estradiol; Gn, gonadotropin; AMH, anti-Mullerian hormone; PROK1, prokineticin 1.

When the two variables were normally distributed, a Pearson correlation analysis was used. When the variables did not conform to the assumption of a normal distribution of bivariate variables, a Spearman's rank correlation analysis was performed. Linear regression was used to analyze the association between the dose of $\mathrm{Gn}$ and the levels of E2 and PROK1. A $\chi^{2}$ test was used for the comparison of frequencies. The area under the receiver operating characteristic (ROC) curve (AUC) was used to evaluate the accuracy of follicular fluid E2, $\mathrm{AMH}$ and PROK1 levels in predicting pregnancy rate. $\mathrm{P}<0.05$ was considered to indicate a statistically significant difference.

\section{Results}

General characteristics of participants. A total of 81 patients met the inclusion criteria and 77 follicular fluid specimens that met the requirements were obtained (Table I).

Comparison between the absolute pregnancy group and absolutenon-pregnancy group. Among 53 patients with embryo transfer, 20 cases exhibited an absolute pregnancy, 27 cases exhibited an absolute non-pregnancy, and 6 cases exhibited a possible pregnancy. Among the 20 cases with absolute pregnancy, 13 had a single fetus and 7 had a double fetus. The concentration of E2 in the follicular fluid from the largest follicles in the absolute pregnancy group $(526419 \pm 289944 \mathrm{pg} / \mathrm{ml})$ was significantly lower compared with the absolute non-pregnancy group $(736085 \pm 261885 \mathrm{pg} / \mathrm{ml} ; \mathrm{t}=-2.742 ; \mathrm{P}=0.012$; Fig. 1A; Table II). The concentrations of PROK1 and AMH in the follicular fluid from the largest follicles in absolute pregnancy group were not significantly different from those in the absolute non-pregnancy group (Fig. 1B and C; Table II). In addition, the concentrations of peripheral blood PROK1 and $\mathrm{AMH}$ in the absolute pregnancy group were not significantly different from those in the absolute non-pregnancy group on the day of egg retrieval (Table II). The fertilization rate of absolute pregnancy group was significantly higher than that of absolute non-pregnancy group $\left(\chi^{2}=5.861 ; \mathrm{P}=0.015\right.$; Table II $)$.

Concentration of E2 in follicular fluid is associated with the $\mathrm{Gn}$ dose. The relationship between $\mathrm{Gn}$ dose and E2 concentration in follicular fluid was analyzed by linear regression analysis. The regression coefficient is -164.865 , constant 942432.470. T statistic value of regression coefficient t-test is $-2.670, \mathrm{r}^{2}=0.087, \mathrm{P}=0.009$ (Fig. 2A). Therefore, regression coefficient can be considered to be significant, 
Table III. Number of retrieved eggs, fertilization rate and good embryo rate in the low and high E2 groups.

\begin{tabular}{lcccr}
\hline Characteristic & $\begin{array}{c}\text { Low E2 group } \\
(\text { mean } \pm \text { SD })\end{array}$ & $\begin{array}{c}\text { High E2 group } \\
(\text { mean } \pm \text { SD })\end{array}$ & $\chi^{2} /$ t-value & P-value \\
\hline No. of retrieved eggs & $9.03 \pm 4.23$ & $8.37 \pm 4.59$ & $0.565^{\mathrm{a}}$ & 0.520 \\
Fertilization rate & $81.99 \%(264 / 322)$ & $80.74 \%(239 / 296)$ & $0.158^{\mathrm{b}}$ & 0.691 \\
Good embryo rate & $70.89 \%(168 / 237)$ & $65.78 \%(148 / 225)$ & $1.393^{\mathrm{b}}$ & 0.238 \\
\hline
\end{tabular}

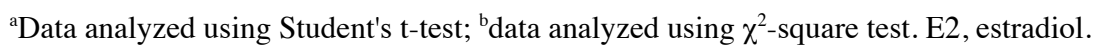
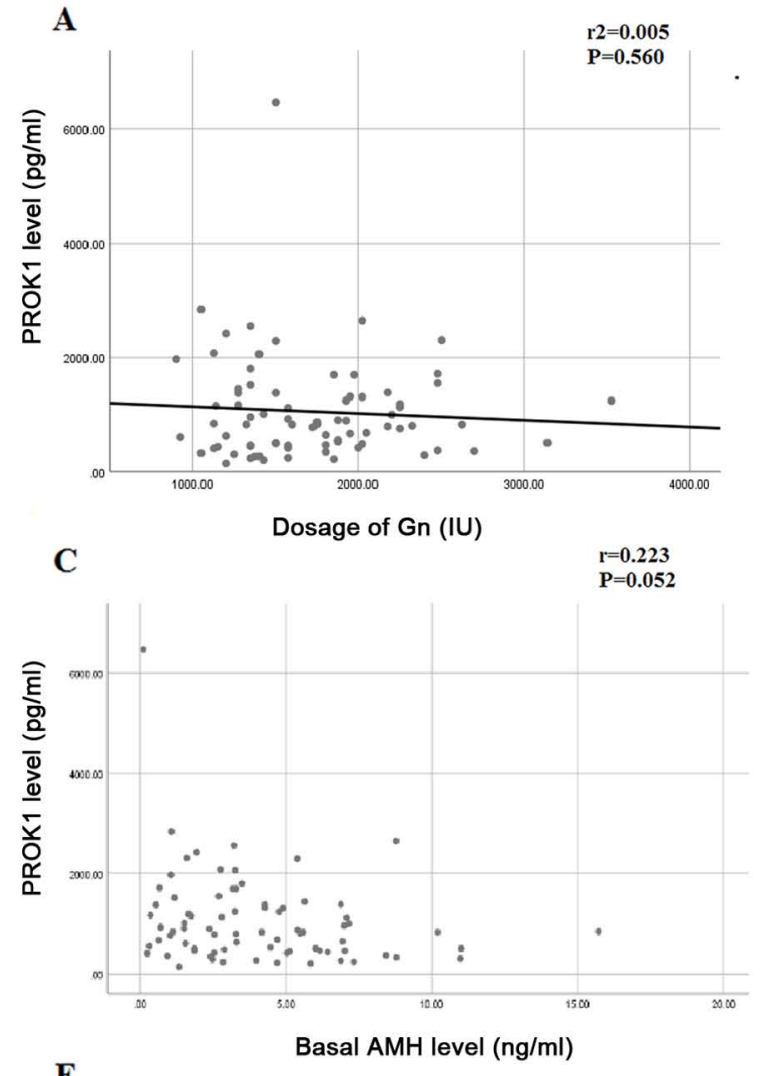

$\mathbf{E}$

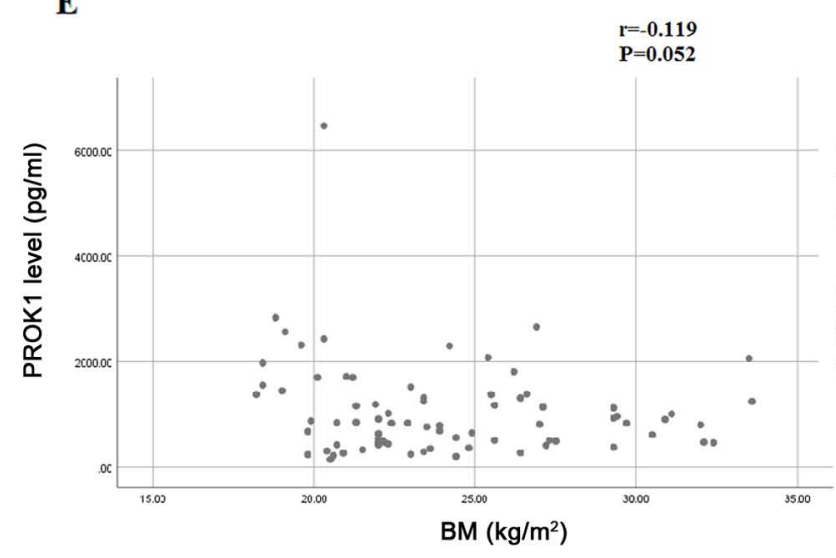

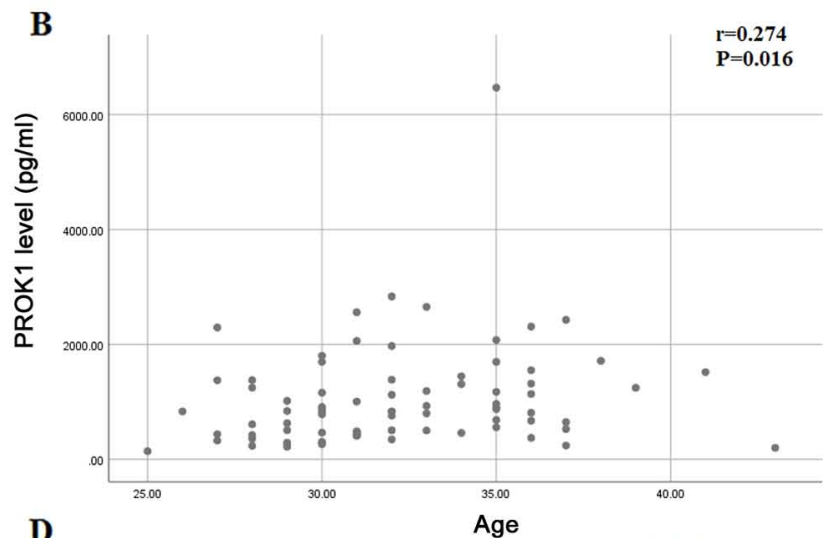

D
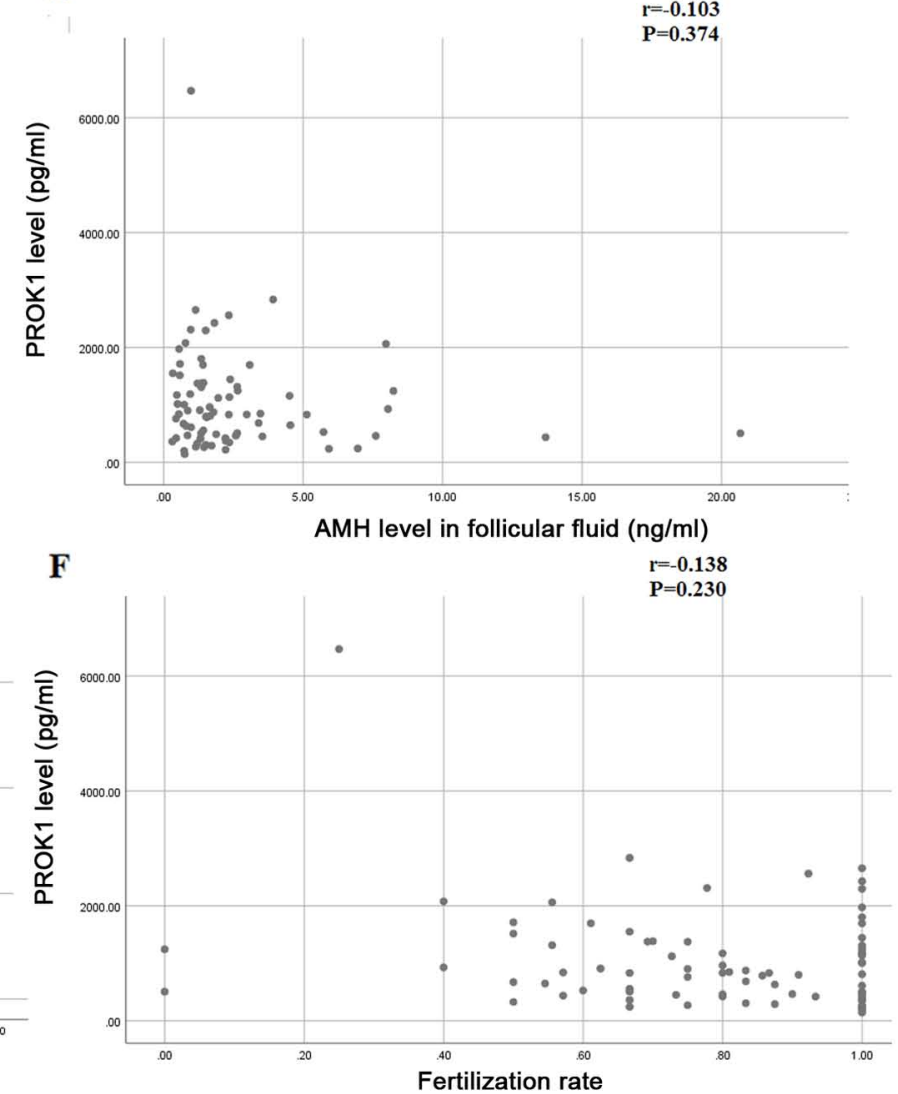

Figure. 3. Correlation between PROK1 in follicular fluid and (A) Gn dosage, (B) age, (C) basal AMH, (D) AMH in follicular fluid, (E) BMI and (F) fertilization rate. PROK1, prokineticin 1; AMH, anti-Mullerian hormone; BMI, body mass index.

from which the linear regression equation can be obtained: $\mathrm{Y}=942432.470-164.865 \mathrm{X}$. To examine if E2 was correlated with other variables, a correlation analysis was performed. The concentration of E2 was not associated with age $(r=0.018$,
$\mathrm{P}=0.878)$, AMH level in follicular fluid $(\mathrm{r}=0.032, \mathrm{P}=0.781)$, PROK1 level in follicular fluid ( $r=-0.019, \mathrm{P}=0.870)$, fertilization rate $(\mathrm{r}=-0.038, \mathrm{P}=0.742)$ or number of usable blastocysts $(\mathrm{r}=-0.109, \mathrm{P}=0.097)$ (Fig. 2B-F). 


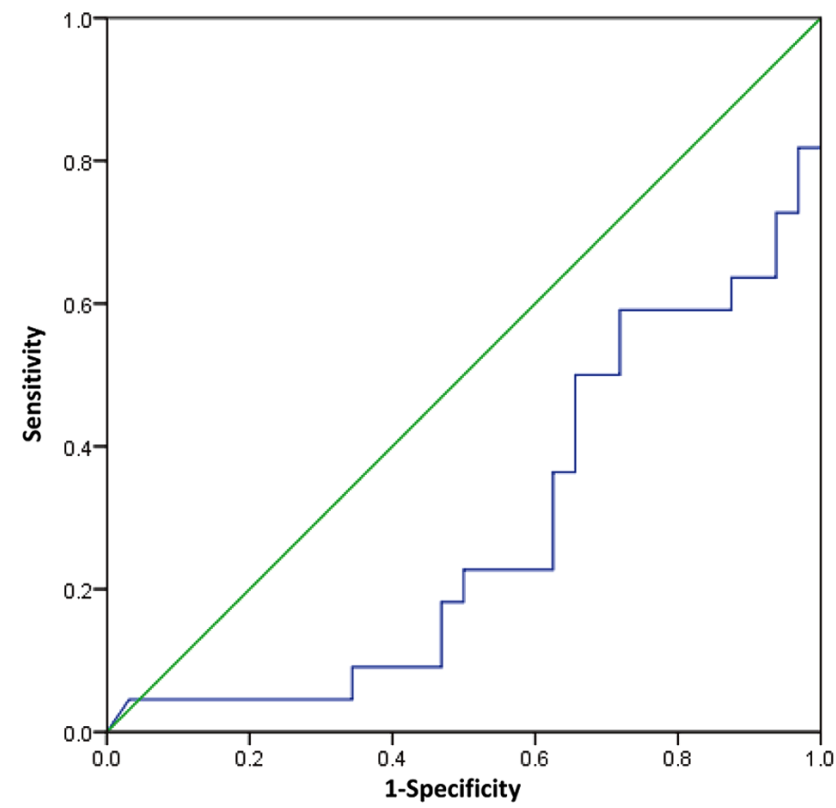

Figure. 4. ROC curve of E2 level in follicular fluid and pregnancy outcome. The ROC and area under curve were calculated. The green line represents the reference line. The blue line represents the E2 results. ROC, receiver operating characteristic curve; E2, estradiol.

Follicular fluid PROK1 level is not correlated with Gn dosage but age. The relationship between the dose of $\mathrm{Gn}$ and the concentration of PROK1 in follicular fluid was analyzed using a linear regression analysis. The dose of $\mathrm{Gn}$ is the independent variable on the $\mathrm{X}$-axis, the concentration of PROK1 in follicular fluid is the dependent variable on the $y$-axis, the determination coefficient, $\mathrm{r}^{2}=0.005$, the regression coefficient is -0.119 , the constant is 1263 , the observation value of $\mathrm{T}$ statistics of regression coefficient $\mathrm{T}$ test is -0.585 , and the $\mathrm{P}$ value of $\mathrm{t}$-test is 0.560 . Therefore, the regression coefficient displayed no significance (Fig. 3A). To examine if PROK1 was correlated with other variables, a correlation analysis was performed. The levels of PROK1 in follicular fluid was correlated with age $(\mathrm{r}=0.274, \mathrm{P}=0.016)$ (Fig. $3 \mathrm{~B})$. There was no significant correlation between PROK1 in follicular fluid and basal AMH ( $r=0.223, \mathrm{P}=0.052)$, AMH in follicular fluid $(\mathrm{r}=-0.103$, $\mathrm{P}=0.374)$, body mass index $(\mathrm{BMI})(\mathrm{r}=-0.119, \mathrm{P}=0.301)$ and fertilization rate $(r=-0.138, P=0.230)$ (Fig. $3 C-F)$

E2 level does not affect the number of retrieved oocytes, fertilization rate and good embryo rate. Using the median of E2 levels in final fertilized follicular fluid $(676610 \mathrm{pg} / \mathrm{ml})$ from the 75 cases as the cut-off point, these cases were divided into the low E2 group ( $\leq 676610 \mathrm{pg} / \mathrm{ml} ; 37$ cases) and high E2 group ( $>676610 \mathrm{pg} / \mathrm{ml} ; 38$ cases). The number of retrieved oocytes, fertilization rate and good embryo rate were not significantly different between the two groups (Table III). The result suggests that E2 level did not affect the number of retrieved oocytes, fertilization rate and good t embryo rate.

E2 level in follicular fluid can predict the outcome of pregnancy. To evaluate the predictive value of E2 level in follicular fluid, a ROC curve was plotted and AUC was calculated. The AUC was 0.283 (95\% CI, 0.144-0.423; P=0.007;
Fig. 4). The AUC was significant which indicates that E2 levels can discriminate between pregnancy outcomes. The optimum cut off value of E2 level in follicular fluid is $689345 \mathrm{pg} / \mathrm{ml}$, which was shown to predict pregnancy outcome with a sensitivity of $27.3 \%$, and specificity of $37.5 \%$.

\section{Discussion}

The concentration of E2 in the largest single follicular fluid $(657425 \pm 275979 \mathrm{pg} / \mathrm{ml})$ of the present study was $10-50$ times greater than has been reported previously $(30,31)$. This may be due to the high number of granulosa cell layers in the largest follicle, which increases E2 secretion (32). Additionally, E2 concentration in peripheral blood on the day of egg retrieval was $1565 \pm 1001 \mathrm{pg} / \mathrm{ml}, 400$ times lower than that in the follicular fluid. The median level of PROK1 in the follicular fluid on the day of egg retrieval was only 21 times greater than that in the peripheral blood, demonstrating that vascular endothelial cells underwent rapid proliferation from luteinized granular cells to well-functioning granular corpus luteum and membranous corpus luteum. PROK1 vasculogenic factor is associated with this aforementioned process, in which PROK1 aggregates in a short amount of time to exhibit an increased PROK1 level compared with the peripheral blood $(29,33)$. By contrast, the median level of AMH in the follicular fluid on the day of egg retrieval was only slightly higher than levels in the peripheral blood. This result may be due to AMH secreted by small antral follicles being the main source of $\mathrm{AMH}$ in the peripheral blood, instead of follicular fluid AMH (34).

E2 level in the follicular fluid of the absolute pregnancy group was demonstrated to be significantly lower than that in the absolute non-pregnancy group, suggesting that high levels of estrogen had a negative effect on the pregnancy outcome. It has been previously reported that high estrogen levels in the peripheral blood can lead to poor pregnancy outcomes by affecting endometrial receptivity $(35,36)$. In the present study, high E2 levels in the follicular fluid exhibited an adverse effect on pregnancy outcome, suggesting that abnormally high estrogen levels might affect the development potential of adjacent oocytes via a variety of autocrine paracrine signaling pathways. One previous study has revealed that E2 concentration in single follicular fluid was negatively correlated with good embryo rate (7). However, the current study failed to demonstrate this correlation (data not shown). The results indicated that a high E2 level in the follicular fluid was negatively associated with $\mathrm{Gn}$ dose. The ROC curve analyzed the diagnostic accuracy of E2 levels to discriminate between absolute pregnancy per transfer and absolute non pregnancy per transfer. In the ROC model, E2 levels were predictive of pregnancy outcome. However, the AUC was $<0.5$ and the sensitivity and specificity of the cut off value was low.

The selection of dominant follicles often requires complex capillary networks, and angiogenesis is closely associated with follicular development (9). In the ovaries, PROK1 not only promotes angiogenesis, but also affects ovarian function $(19,29)$. However, its specific roles have not yet been clearly determined. A previous study has demonstrated that PROK1 secretion in the follicular fluid is significantly different between pregnant and non-pregnant patient groups (19); however, contradictory results have 
also been reported (21). In the present study, no significant differences were observed between PROK1 expression in the follicular fluid of the pregnant and non-pregnant group. The promoter of the PROK1 gene has a cAMP response element (CRE) binding site (20). It has been previously reported that AMH inhibited the binding of follicle-stimulating hormone to follicle-stimulating hormone receptor through autocrine paracrine and affects the transcription of estrogen synthetase (37). The results in the present study revealed that PROK1 expression and AMH in the follicular fluid exhibited no correlation. The level of PROK1 in follicular fluid was correlated with age but not correlated with basal AMH. The level of PROK1 in follicular fluid of older people showed an increasing trend However, this possible correlation between the two requires further study.

In the present study, no significant differences were exhibited in the baseline characteristics between the absolute pregnancy group and the absolute non-pregnancy group, suggesting that the two groups were comparable. However, the limitation of the present study is that a small number of cases was used for analysis, which may affect the results. Additionally, the upper limit of the electrochemiluminescence reagent for the determination of E2 concentration was $3,000 \mathrm{pg}$, and some samples required dilution 400 times, which may have also influenced the results.

In conclusion, the present study demonstrated that E2 level in follicular fluid is a better predictor of the outcome of IVF-ET compared with AMH or PROK1 level determination.

\section{Acknowledgements}

The authors wish to thank Dr Jiangfeng Lv from Clinical Laboratory of Jinan People's Hospital (Jinan, China) and Dr Xiumei Yu from Affiliated Yuhuangding Hospital of Qingdao University (Yantai, China) for their help in the hormone tests.

\section{Funding}

The current study was supported by the National Natural Science Foundation of China (grant. no. 81671416).

\section{Availability of data and materials}

The datasets used and/or analyzed during the current study are available from the corresponding author on reasonable request.

\section{Authors' contributions}

Each author believes that the manuscript represents honest work. YL and $\mathrm{CH}$ collaborated to design the study. YL and $\mathrm{XH}$ were responsible for performing experiments. YL and SD analyzed the data. All authors collaborated to interpret results and develop the manuscript. All authors read and approved the final manuscript.

\section{Ethics approval and consent to participate}

All procedures performed in the current study were approved by the Ethics Committee of Affiliated Yuhuangding Hospital of Qingdao University. Written informed consent was obtained from all patients or their families.

\section{Patient consent for publication}

Not applicable.

\section{Competing interests}

The authors declare that they have no competing interests.

\section{References}

1. Durán Reyes G, Rosales AM and Hicks Gómez JJ: Participation of the follicular fluid in follicular development, oocyte maturation and spermatic function. Ginecol Obstet Mex 65: 349-356, 1997 (In Spanish).

2. Hammadeh ME, Ertan AK, Zeppezauer M, Baltes S, Georg T, Rosenbaum P and Schmidt W: Immunoglobulins and cytokines level in follicular fluid in relation to etiology of infertility and their relevance to IVF outcome. Am J Reprod Immunol 47: 82-90, 2002.

3. Lédée N, Petitbarat M, Rahmati M, Dubanchet S, Chaouat G, Sandra O, Perrier-d'Hauterive S, Munaut C and Foidart JM: New pre-conception immune biomarkers for clinical practice: Interleukin-18, interleukin-15 and TWEAK on the endometrial side, G-CSF on the follicular side. J Reprod Immunol 88: 118-123, 2011.

4. Vercammen M, Verloes A, Haentjens P and Van de Velde H: Can soluble human leucocyte antigen-G predict successful pregnancy in assisted reproductive technology? Curr Opin Obstet Gynecol 21: 285-290, 2009.

5. Hammadeh ME, Fischer-Hammadeh C, Amer AS, Rosenbaum P and Schmidt W: Relationship between cytokine concentration in serum and preovulatory follicular fluid and in vitro fertilization/intracytoplasmic sperm injection outcome. Chem Immunol Allergy 88: 80-97, 2005.

6. Ovayolu A, Özdamar Ö, Gün İ, Arslanbuğa CY, Kutlu T, Tunalı G and Uluhan R: The assesment of follicular fluid presepsin levels in poor ovarian responder womenandits relationship with the reproductive outcomes. Int J Clin Exp Med 8: 9961-9966, 2015.

7. Mehta BN, Chimote MN, Chimote NN, Nath NM and Chimote NM: Follicular-fluid anti-Mullerian hormone (FF $\mathrm{AMH}$ ) is a plausible biochemical indicator of functional viability of oocyte in conventional in vitro fertilization (IVF) cycles. J Hum Reprod Sci 6: 99-105, 2013.

8. Sallam HN, Sallam NH and Sallam SH: Non-invasive methods for embryo selection. Facts Views Vis Obgyn 8: 87-100, 2016.

9. Koo YA, Lee B, Park HJ, Choi J, Lee E and Choi D: Altered vascular endothelial growth factor expression during $\mathrm{GnRH}$ antagonist protocol in women of reproductive age with normal baseline hormone profiles. Fertil Steril 91: 744-748, 2009.

10. Li M, Bullock CM, Knauer DJ, Ehlert FJ and Zhou QY: Identification of two prokineticin cDNAs: Recombinant proteins potently contract gastrointestinal smooth muscle. Mol Pharmacol 59: 692-698, 2001.

11. Matsumoto S, Yamazaki C, Masumoto KH, Nagano M, Naito M, Soga T, Hiyama H, Matsumoto M, Takasaki J, Kamohara M, et al: Abnormal development of the olfactory bulb and reproductive system in mice lacking prokineticin receptor PKR2. Proc Natl Acad Sci USA 103: 4140-4145, 2006.

12. Tu LH, Yu LL, Xiong CL and Zhang HP: Potential role of prokineticin 2 in experimental varicoceleinduced rat testes. Urology 80: 952.e15-19, 2012.

13. Kim KH, Oh DS, Jeong JH, Shin BS, Joo BS and Lee KS: Follicular blood flow is a better predictor of the outcome of in vitro fertilization-embryo transfer than follicular fluid vascular endothelial growth factor and nitric oxide concentrations. Fertil Steril 82: 586-592, 2004.

14. LeCouter J, Kowalski J, Foster J, Hass P, Zhang Z, Dillard-Telm L, Frantz G, Rangell L, DeGuzman L, Keller GA, et al: Identification of an angiogenic mitogen selective for endocrine gland endothelium. Nature 412: 877-884, 2001.

15. Lin R, LeCouter J, Kowalski J and Ferrara N: Characterization of endocrine gland-derived vascular endothelial growth factor signaling in adrenal cortex capillary endothelial cells. J Biol Chem 277: 8724-8729, 2002. 
16. Kisliouk T, Friedman A, Klipper E, Zhou QY, Schams D, Alfaidy $\mathrm{N}$ and Meidan R: Expression pattern of prokineticin 1 and its receptors in bovine ovaries during the estrous cycle: Involvement in corpus luteum regression and follicular atresia. Biol Reprod 76: 749-758, 2007.

17. Lee KF, Lee YL, Chan RW, Cheong AW, Ng EH, Ho PC and Yeung WS: Up-regulation of endocrine gland-derived vascular endothelial growth factor but not vascular endothelial growth factor in human ectopic endometriotic tissue. Fertil Steril 93: 1052-1060, 2010.

18. Kisliouk T, Levy N, Hurwitz A and Meidan R: Presence and regulation of endocrine gland vascular endothelial growth factor/prokineticin-1 and its receptors in ovarian cells. J Clin Endocrinol Metab 88: 3700-3707, 2003

19. Alfaidy N, Hoffmann P, Gillois P, Gueniffey A, Lebayle C, Garçin H, Thomas-Cadi C, Bessonnat J, Coutton C, Villaret L, et al: PROK1 level in the follicular microenvironment: A new noninvasive predictive biomarker of embryo implantation. J Clin Endocrinol Metab 101: 435-444, 2016.

20. Brouillet S, Hoffmann P, Chauvet S, Salomon A, Chamboredon S, Sergent F, Benharouga M, Feige JJ and Alfaidy N: Revisiting the role of hCG: New regulation of the angiogenic factor EG-VEGF and its receptors. Cell Mol Life Sci 69: 1537-1550, 2012.

21. Gao MZ, Zhao XM, Lin Y, Sun ZG and Zhang HQ: Effects of EG-VEGF, VEGF and TGF- $\beta 1$ on pregnancy outcome in patients undergoing IVF-ET treatment. J Assist Reprod Genet 29: 1091-1096, 2012

22. Visser JA and Themmen AP: Anti-Müllerian hormone and folliculogenesis. Mol Cell Endocrinol 234: 81-86, 2005.

23. Fanchin R, Louafi N, Méndez Lozano DH, Frydman N, Frydman R and Taieb J: Per-follicle measurements indicate that anti-müllerian hormone secretion is modulated by the extent of follicular development and luteinization and may reflect qualitatively the ovarian follicular status. Fertil Steril 84: 167-173, 2005

24. Qin Y, Zhao Z, Sun M, Geng L, Che L and Chen ZJ: Association of basal serum testosterone levels with ovarian response and in vitro fertilization outcome. Reprod Biol Endocrinol 9: 9, 2011.

25. Hassan AMA, Kotb MMM, AwadAllah AMA, Shehata NAA and Wahba A: Follicular sensitivity index (FSI): A novel tool to predict clinical pregnancy rate in IVF/ICSI cycles. J Assist Reprod Genet 34: 1317-1324, 2017.

26. La Marca A, Grisendi V, Giulini S, Argento C, Tirelli A, Dondi G, Papaleo E and Volpe A: Individualization of the FSH starting dose in IVF/ICSI cycles using the antral follicle count. J Ovarian Res 6: 11, 2013

27. Kolibianakis EM, Venetis CA, Kalogeropoulou L,Papanikolaou E and Tarlatzis BC: Fixed versus flexible gonadotropin-releasing hormone antagonist administration in in vitro fertilization: A randomized controlled trial. Fertil Steril 95: 558-562, 2011.
28. Huang X, Hao C, Shen X, Liu X, Shan Y, Zhang Y and Chen L: Differences in the transcriptional profiles of human cumulus cells isolated from MI and MII oocytes of patients with polycystic ovary syndrome. Reproduction 145: 597-608, 2013.

29. Vural F, Vural B, Doğer E, Çakıroğlu Y and Çekmen M: Perifollicular blood flow and its relationship with endometrial vascularity, follicular fluid EG-VEGF, IGF-1, and inhibin-a levels and IVF outcomes. J Assist Reprod Genet 33: 1355-1362, 2016.

30. Ruiz de Assín R, Clavero A, Gonzalvo MC, Ramírez JP, Zamora S, Fernández A, Martínez L and Castilla JA: Comparison of methods to determine the assigned value in an external quality control programme for embryo evaluation. Reprod Biomed Online 19: 824-829, 2009.

31. Nardo LG, Gelbaya TA, Wilkinson H, Roberts SA, Yates A, Pemberton $\mathrm{P}$ and Laing I: Circulating basal anti-Mullerian hormone levels as predictor of ovarian response in women undergoing ovarian stimulation for in vitro fertilization. Fertil Steril 92: 1586-1593, 2009

32. Rodgers RJ and Irving-Rodgers HF: Formation of the ovarian follicular antrum and follicular fluid. Biol Reprod 82: 1021-1029, 2010.

33. Fraser HM, Bell J, Wilson H, Taylor PD, Morgan K, Anderson RA and Duncan WC: Localization and quantification of cyclic changes in the expression of endocrine gland vascular endothelial growth factor in the human corpus luteum. J Clin Endocrinol Metab 90: 427-434, 2005.

34. Durlinger AL, Visser JA and Themmen AP: Regulation of ovarian function: The role of anti-Mullerian hormone. Reproduction 124: 601-609, 2002

35. Kosmas IP, Kolibianakis EM and Devroey P: Association of estradiol levels on the day of hCG administration and pregnancy achievement in IVF: A systematic review. Hum Reprod 19: 2446-2453, 2004.

36. Yu Ng EH, Yeung WS, Yee Lan Lau E, So WW and Ho PC: High serum oestradiol concentrations in fresh IVF cycles do not impair implantation and pregnancy rates in subsequent frozen-thawed embryo transfer cycles. Hum Reprod 15: 250-255, 2000.

37. Kanakkaparambil R, Singh R, Li D, Webb R and Sinclair KD: B-vitamin and homocysteine status determines ovarian response to gonadotropin treatment in sheep. Biol Reprod 80: 743-752, 2009.

(i) $(-)$ This work is licensed under a Creative Commons Attribution-NonCommercial-NoDerivatives 4.0 International (CC BY-NC-ND 4.0) License. 